\title{
Efficacy of non-invasive and invasive respiratory management strategies in adult patients with acute hypoxaemic respiratory failure: a systematic review and network meta-analysis
}

Masaaki Sakuraya ${ }^{1 *}\left(\mathbb{0}\right.$, Hiromu Okano ${ }^{2} \mathbb{B}$, Tomoyuki Masuyama ${ }^{3}$, Shunsuke Kimata ${ }^{4}$ and Satoshi Hokari ${ }^{5}(\mathbb{0}$

\begin{abstract}
Background: Although non-invasive respiratory management strategies have been implemented to avoid intubation, patients with de novo acute hypoxaemic respiratory failure (AHRF) are high risk of treatment failure. In the previous meta-analyses, the effect of non-invasive ventilation was not evaluated according to ventilation modes in those patients. Furthermore, no meta-analyses comparing non-invasive respiratory management strategies with invasive mechanical ventilation (IMV) have been reported. We performed a network meta-analysis to compare the efficacy of non-invasive ventilation according to ventilation modes with high-flow nasal oxygen (HFNO), standard oxygen therapy (SOT), and IMV in adult patients with AHRF.
\end{abstract}

Methods: The Cochrane Central Register of Controlled Trials, MEDLINE, EMBASE, and Ichushi databases were searched. Studies including adults with AHRF and randomized controlled trials (RCTs) comparing two different respiratory management strategies (continuous positive airway pressure (CPAP), pressure support ventilation (PSV), HFNO, SOT, or IMV) were reviewed.

Results: We included 25 RCTs (3,302 participants: 27 comparisons). Using SOT as the reference, CPAP (risk ratio [RR] 0.55; 95\% confidence interval [CI] 0.31-0.95; very low certainty) was associated significantly with a lower risk of mortality. Compared with SOT, PSV (RR 0.81; $95 \% \mathrm{Cl} 0.62-1.06$; low certainty) and HFNO (RR 0.90; 95\% Cl 0.65-1.25; very low certainty) were not associated with a significantly lower risk of mortality. Compared with IMV, no non-invasive respiratory management was associated with a significantly lower risk of mortality, although all certainties of evidence were very low. The probability of being best in reducing short-term mortality among all possible interventions was higher for CPAP, followed by PSV and HFNO; IMV and SOT were tied for the worst (surface under the cumulative ranking curve value: 93.2, 65.0, 44.1, 23.9, and 23.9, respectively).

Conclusions: When performing non-invasive ventilation among patients with de novo AHRF, it is important to avoid excessive tidal volume and lung injury. Although pressure support is needed for some of these patients, it should be applied with caution because this may lead to excessive tidal volume and lung injury.

*Correspondence: masaaki.sakuraya@gmail.com

1 Department of Emergency and Intensive Care Medicine, JA Hiroshima General Hospital, Jigozen 1-3-3, Hatsukaichi, Hiroshima 738-8503, Japan

Full list of author information is available at the end of the article

(c) The Author(s) 2021. Open Access This article is licensed under a Creative Commons Attribution 4.0 International License, which permits use, sharing, adaptation, distribution and reproduction in any medium or format, as long as you give appropriate credit to the original author(s) and the source, provide a link to the Creative Commons licence, and indicate if changes were made. The images or other third party material in this article are included in the article's Creative Commons licence, unless indicated otherwise in a credit line to the material. If material is not included in the article's Creative Commons licence and your intended use is not permitted by statutory regulation or exceeds the permitted use, you will need to obtain permission directly from the copyright holder. To view a copy of this licence, visit http://creativecommons.org/licenses/by/4.0/. The Creative Commons Public Domain Dedication waiver (http://creativecommons.org/publicdomain/zero/1.0/) applies to the data made available in this article, unless otherwise stated in a credit line to the data. 
Trial registration protocols.io (Protocol integer ID 49375, April 23, 2021). https://doi.org/10.17504/protocols.io.buf7ntrn.

Keywords: Acute hypoxaemic respiratory failure, Continuous positive airway pressure, High-flow nasal oxygen, Network meta-analysis, Non-invasive ventilation

\section{Background}

Acute hypoxaemic respiratory failure (AHRF) is the most common cause of intensive care unit (ICU) admission among adult patients, with a hospital mortality rate of approximately $30 \%$ [1]. Non-invasive respiratory management has been investigated widely among patients with AHRF. Non-invasive ventilation is recommended to reduce the risk of endotracheal intubation and mortality in patients with AHRF, especially due to cardiopulmonary oedema [2]. Compared with standard oxygen therapy (SOT), high-flow nasal oxygen (HFNO) is also a preferable option for patients with AHRF [3].

While non-invasive ventilation has been reported to be used in $15 \%$ of patients with acute respiratory distress syndrome (ARDS), it may be associated with higher ICU mortality, especially in patients with severe hypoxaemia [4]. A definite diagnosis of ARDS [5] may be difficult or impossible before the implementation of respiratory management strategies, because the precise measurement of the actual inspired fraction of oxygen may be unavailable and the positive end-expiratory pressure (PEEP) not used. Furthermore, when implementing noninvasive respiratory management strategies in patients with AHRF, we need to consider the cause of the respiratory failure, especially whether it was an established disease for efficacy of non-invasive ventilation including cardiopulmonary oedema or not. De novo AHRF refers to AHRF that occurs without any prior chronic respiratory diseases [6]. Most patients in this category have pneumonia or ARDS with neither heart failure nor chronic obstructive pulmonary disease (COPD). Noninvasive ventilation is not recommended in patients with de novo AHRF [6], and the efficacy of the HFNO has not been consistent among these patients $[7,8]$.

Excessive tidal volume has been reported to be associated with treatment failure in patients with AHRF [9], and treatment failure has been shown to increase hospital mortality [4]. Although pressure support is needed for hypercapnic respiratory failure, the role of pressure support is unclear in patients with de novo AHRF. Furthermore, there may the possibility of increasing tidal volume and lung injury. A systematic review and network meta-analysis (NMA) was performed recently to evaluate the efficacy of non-invasive respiratory management strategies in adult patients with AHRF, compared with SOT [10]. This NMA divided non-invasive ventilation into two categories: those using a face mask and those using a helmet interface, and showed that helmet non-invasive ventilation was the most effective method to reduce the risk of all-cause mortality and endotracheal intubation. Moreover, continuous positive airway pressure (CPAP) was used as a non-invasive ventilation mode along with helmet non-invasive ventilation in most randomized controlled trials (RCTs) included in this NMA. However, no meta-analyses have evaluated the efficacy of non-invasive ventilation according ventilation mode among patients with AHRF. Furthermore, in the previous NMA, non-invasive respiratory management strategies were not compared with invasive mechanical ventilation (IMV). Although non-invasive respiratory management strategies have been used to avoid complications of IMV and improve clinical outcomes, few meta-analyses comparing noninvasive respiratory management strategies with IMV have been reported.

When performing non-invasive ventilation in patients with AHRF, both PEEP and pressure support are expected to improve oxygenation. However, tidal recruitment provided by pressure support may contribute to not only oxygenation improvement but also lung injury. In this study, we hypothesized that CPAP was the most effective strategy for reducing mortality and endotracheal intubation in patients with de novo AHRF. We performed an NMA to compare the efficacy of non-invasive ventilation according to the ventilation modes with HFNO, SOT, and IMV in adult patients with AHRF.

\section{Methods}

\section{Protocol and registration}

This systematic review was designed according to the Preferred Reporting Items for Systematic review and Meta-Analyses extension statement for reviews incorporating network meta-analyses (details shown in Additional file 1: Table S1) [11], and the protocol was registered at protocols.io (Protocol integer ID 49,375) [12].

\section{Eligibility criteria \\ Type of studies}

We included all the RCTs reported in the publication status (published, unpublished, and academic abstracts). Randomized crossover, cluster-randomized, or quasi-experimental trials were excluded. 


\section{Type of participants}

This review included adults (age $\geq 18$ years) with AHRF, defined by any of the following criteria: new onset ( $<7$ days) of clinical signs (e.g. tachypnoea, increased work of breathing); radiologic signs (unilateral or bilateral chest X-ray opacities); and hypoxaemia. Hypoxaemia was defined as the ratio of arterial oxygen partial pressure to fractional inspired oxygen (P/F ratio) below $300 \mathrm{cmH}_{2} \mathrm{O}$, arterial or percutaneous oxygen saturation $<94 \%$ in room air, or partial pressure of arterial oxygen $<60 \mathrm{mmHg}$ in room air or $<80 \mathrm{mmHg}$ with oxygen. The current meta-analysis excluded studies in which more than half of the patients in whom there was the presence of cardiopulmonary oedema, acute exacerbation of COPD or acute exacerbation of asthma, hypercapnia (e.g. $>50 \mathrm{mmHg}$ ), post-extubation respiratory failure, post-surgical status, trauma, do-not-resuscitate orders, or limited intervention in the emergency department or prehospital care. The rationale for excluding studies that had a primary enrolment of these patients was based on the established efficacy of non-invasive ventilation $[6,13]$, the possibility of increasing non-pulmonary causes (e.g. airway problem, atelectasis due to pain or surgical procedures, and chest wall instability), and uncertain effects due to limited resources.

\section{Types of interventions and comparators}

We included RCTs that compared at least two of the following five methods:

1. SOT: Low-flow nasal cannula, face mask, and venturi mask (with no limit on the flow rate).

2. CPAP: CPAP was used as an initial non-invasive ventilation mode. The type of interface, duration of ventilation, management during the non-invasive ventilation interval, and methods of weaning were not limited.

3. PSV: Pressure support ventilation (PSV), pressure control ventilation, bi-level positive airway pressure, or spontaneous/timed were used initial non-invasive ventilation modes. The type of interface, duration of ventilation, management during the non-invasive ventilation interval, and methods of weaning were not limited.

4. HFNO: The flow rates and fractions of inspired oxygen were not limited.

5. IMV: Mechanical ventilation via endotracheal intubation not tracheostomy with or without a lung-protective strategy.

\section{Type of outcomes}

The primary outcomes were short-term mortality measured at the longest time point reported in the follow-up period ( $<100$ days), ICU discharge, and hospital discharge. The secondary outcome was the incidence of intubation during the ICU stay.

\section{Information sources}

We searched the following databases for eligible trials: The Cochrane Central Register of Controlled Trials; MEDLINE via PubMed; EMBASE; and Ichushi, a database of Japanese research papers. Using a manual search, we also included studies and all systematic reviews on clinical questions about non-invasive respiratory management strategies in the Japanese ARDS Clinical Practice guideline.

\section{Search}

We used the terms 'ARDS, 'adult respiratory distress syndrome', 'respiratory failure', or 'acute lung injury' AND 'non-invasive ventilation', 'NIV', 'oxygen therapy', 'HFNO', or 'high-flow therapy' in searches performed in June 2020 (details in Additional file 1: Table S2). A literature search was also performed from the inception of the database up to May 30, 2021. Search terms included 'pediatric' or 'neonate' and we included articles written only in English and Japanese, because the systematic review was performed originally for clinical questions in the Japanese ARDS Clinical Practice guideline for adults and pediatrics. During the screening process, we excluded studies with pediatric patients.

\section{Study selection}

Two of the five physicians (HO, TM, SH, SK, and MS) screened the title and abstract or the full text for relevant studies during the first and second screenings, respectively, and extracted data from the included studies into standardized data forms, independently. Disagreements, if any, were resolved through discussions with one of five physicians who did not screen that particular study; the original authors were contacted for clarification, as required. For abstract-only studies that could not be evaluated for eligibility based on our review criteria, we attempted to contact the authors. Discrepancies between two reviewers were resolved through mutual discussions or discussions with a third reviewer, as needed.

\section{Data collection process}

After identifying studies in the second screening, data were extracted from each study by the reviewers (HO, TM, SH, SK, and MS) using two tools: the 
Cochrane Data Collection Form (RCTs only) [14] and Review Manager software (RevMan version 5.4.1, The Cochrane Collaboration, 2020) [15]. For cases with unknown data, the authors were contacted.

\section{Data items}

We extracted the following study characteristics:

1. Methods: study design, total study duration, number and locations of study centres, study setting, withdrawals, date of study initiation, and funding sources.

2. Participants: number, mean age, age range, sex, severity of condition, diagnostic criteria, and inclusion/exclusion criteria.

3. Interventions: treatment approaches and comparison methods.

4. Outcomes: primary and secondary outcomes that were specified and collected and the timepoints reported.

\section{Geometry of the network}

Network plots were constructed to determine the number of studies and patients included in this meta-analysis. We demonstrated the network geometry that presented the nodes as interventions and each head-to-head direct comparison as lines connecting these nodes. The size of the nodes was proportional to the number of participants in each node. The thickness of the connecting line was proportional to the number of randomized clinical trials in each comparison.

\section{Risk of bias within individual studies}

The risk of bias of outcomes in the included studies was assessed independently by two of the five authors ( $\mathrm{HO}$, TM, SH, SK, and MS) using a modified version of the Cochrane 'Risk of Bias' instrument [16]. They assessed the overall risk of bias as the worst in any of the following domains: from the randomisation process, deviations from intended interventions, missing outcome data, measurement of the outcomes, and selection of the reported results. The risk of each bias was graded as 'low risk of bias', 'some concerns', or 'high risk of bias'. Discrepancies between two reviewers were resolved through discussion among themselves or with a third reviewer, as necessary.

\section{Planned methods of analyses Direct comparison meta-analysis}

A pair-wise meta-analysis was performed using RevMan 5.3 (RevMan 2014) [15]. Forest plots were used for the meta-analysis, and the effect size was expressed as risk ratio (RRs) with $95 \%$ confidence intervals (CIs) for the categorical data. The outcome measures were pooled using a random-effects model for the measure of studyspecific effects. For all the analyses, a two-sided $P<0.05$ was considered to be statistically significant.

\section{Network comparison meta-analysis}

Data synthesis An NMA was performed using a frequentist approach with multivariate random-effects meta-analysis with the mvmeta command in Stata 15.1 (StataCorp LLC, College Station, TX, USA).

The network meta command allowed us to fit consistency models and estimate network RRs for each treatment strategy based on both direct and indirect comparisons [17]. We constructed forest plots of the RRs with $95 \%$ CIs for each treatment strategy in the network.

Ranking Ranking plots (rankograms) were constructed based on the probability that a given treatment had the highest event rate for each outcome. The surface under the cumulative ranking curve (SUCRA), which is a simple transformation of the mean rank, was used to determine the treatment hierarchy [18]. Higher values of the SUCRA statistic, which range from 0 to $100 \%$, increase the likelihood that a therapy is ranked among the best in an NMA [19].

\section{Assessment of inconsistency}

Study heterogeneity among trials for each outcome was assessed by inspecting the forest plots visually and using the $\mathrm{I}^{2}$ statistic to quantify any inconsistencies [20]. Publication bias was assessed visually using a funnel plot [19].

Coherence in NMA referred to consistency in the estimates of treatment effects between direct and indirect comparisons [21]. For each pair-wise comparison, we assessed the coherence using a node-splitting method [22]. We also examined coherence globally across the network using the Wald Chi-square test, obtained by fitting the inconsistency model [17].

\section{Grades of Recommendation, Assessment, Development and Evaluation Working Group (GRADE) assessments of the certainty of evidence for each network comparison}

To assess the certainty of evidence for direct comparisons, we used a standard GRADE methodology [23-25]. We rated down for risk of bias, indirectness, inconsistency, and publication bias but did not rate down for imprecision because this occurred at a later step [26, 27]. For indirect comparisons, we started with the lowest certainty of evidence for the contributing direct comparisons and then rated down if there was substantial intransitivity. The transitivity assumption underlying the NMA was evaluated by comparing the distribution of clinical and methodological variables that could act as effect 
modifiers across treatment comparisons. We assessed the certainty in each network comparison considering the highest certainty of evidence between the direct and indirect evidence [28]; the network estimate was rated subsequently taking into account the imprecision and incoherence $[29,30]$.

\section{Additional analysis}

A pre-planned sensitivity analysis, which excluded studies using a helmet interface, was performed to assess the robustness of the findings. In addition, we performed post hoc sensitivity analyses to explore the sources of significant incoherence that were present for the primary outcome. The post hoc sensitivity analyses were performed as follows: according to oxygenation (mean P/F ratio $>150$ or $\leq 150$ ) and immunocompromised status; excluding studies that enrolled any patients with COPD or cardiopulmonary oedema and studies with high risk of bias; and including studies that reported short-term mortality within 30 days and studies published after 2000 .

\section{Results}

\section{Study selection}

The search strategy identified 14,263 records, including 25 RCTs (3302 participants; range 30-776 participants) that were eligible for inclusion (Fig. 1).

\section{Presentation of network structure and summary of network geometry}

The included trials evaluated five different interventions, and these included five of 10 potential head-to-head comparisons for short-term mortality as well as four different interventions and four of six potential head-tohead comparisons for intubation. Specifically, nine trials compared PSV with SOT [31-39], five trials compared CPAP with SOT [40-44], five trials compared HFNO with SOT $[8,45-48]$, three trials compared PSV with IMV [49-51], and two trials compared PSV with HFNO $[52,53]$ (Table 1; Fig. 2). In addition, a three-group study directly compared PSV with HFNO and SOT [7]. No studies compared CPAP or HFNO with IMV. There were 27 comparisons in 25 RCTs.

\section{Study characteristics and risk of bias assessment}

The participants, interventions, comparisons, outcomes, and cohort characteristics of the included trials are shown in Table 1 and Additional file 1: Table S3. The mean age at randomisation ranged from 46 to 73 years, the mean $\mathrm{P} / \mathrm{F}$ ratio was predominantly $<200$ (16 trials [64.0\%]) [7, 8, 32, 34, 35, 38, 40, 41, 44, 45, 47, 49-53], and the mean partial pressure of arterial carbon dioxide $\left(\mathrm{PaCO}_{2}\right)$ was $>50 \mathrm{mmHg}$ in one trial $(4.0 \%)$ [41]. Nine trials $(36.0 \%)$ included immunocompromised patients
[8, 34, 36-38, 43-45, 48]. Community-acquired pneumonia was the most common cause of AHRF in 14 trials (56.0\%) [7, 8, 31, 32, 34-36, 38-40, 42, 44, 47, 50]. Helmet interfaces were used in three of five trials comparing CPAP with SOT [42-44] and in a trial comparing PSV with HFNO [53]. In two of three trials comparing PSV with IMV $[49,50]$, target tidal volume was set at $8 \mathrm{ml} / \mathrm{kg}$ (of the predicted body weight) or more for mechanically ventilated patients.

\section{Non-invasive respiratory management strategies and risk of short-term mortality}

Twenty-three trials (3169 patients) were included in the short-term mortality analysis. The pair-wise comparisons are shown in Additional file 1: Figure S1. The risk of bias was determined to be high for the outcome of mortality in six (26.1\%) trials (Additional file 1: Table S4). We did not rate down due to publication bias (the funnel plot shown in Additional file 1: Figure S2); however, we assessed that the risk of bias was substantial between CPAP and SOT and; therefore, rated down. We also rated down considering inconsistencies in the direct comparisons of CPAP versus SOT, PSV versus IMV, and PSV versus HFNO (Additional file 1: Table S5). Incoherence between the direct and indirect RRs was observed for the comparisons of HFNO versus SOT, PSV versus SOT, and PSV versus HFNO. We also identified a significant global incoherence across the network.

Using SOT as the reference, CPAP (RR 0.55 [95\% CI $0.31-0.95]$; risk difference $[R D]-0.14[95 \% \mathrm{CI}-0.21$ to -0.02 ]; very low certainty) was associated significantly with a lower risk of mortality (Fig. 3). Compared with SOT, PSV (RR 0.81 [95\% CI 0.62-1.06]; RD, - 0.06 [95\% $\mathrm{CI}-0.11$ to 0.02 ]; low certainty) and HFNO (RR, 0.90 [95\% CI 0.65-1.25]; RD -0.03 [95\% CI -0.11 to 0.08 ]; very low certainty) were not associated with a statistically significant lower risk of mortality.

Compared with IMV, CPAP (RR 0.51 [95\% CI 0.221.15]; $\mathrm{RD}-0.15$ [95\% CI -0.23 to 0.05 ]; very low certainty), PSV (RR 0.75 [95\% CI 0.43-1.30]; RD - 0.08 [95\% CI -0.17 to 0.09 ]; very low certainty), and HFNO (RR 0.83 [95\% CI 0.43-1.62]; RD-0.05 [95\% CI-0.17 to 0.19 ]; very low certainty) were not associated with a statistically significant lower risk of mortality, and all certainties of evidence were very low. Although CPAP tended to be associated with a lower risk of mortality, there were no significant differences among the noninvasive respiratory management strategies. The probability of being the best in reducing short-term mortality among all possible interventions was higher for CPAP, followed by PSV and HFNO; IMV and SOT tied for the worst (Table 2; Additional file 1: Figure S3). 


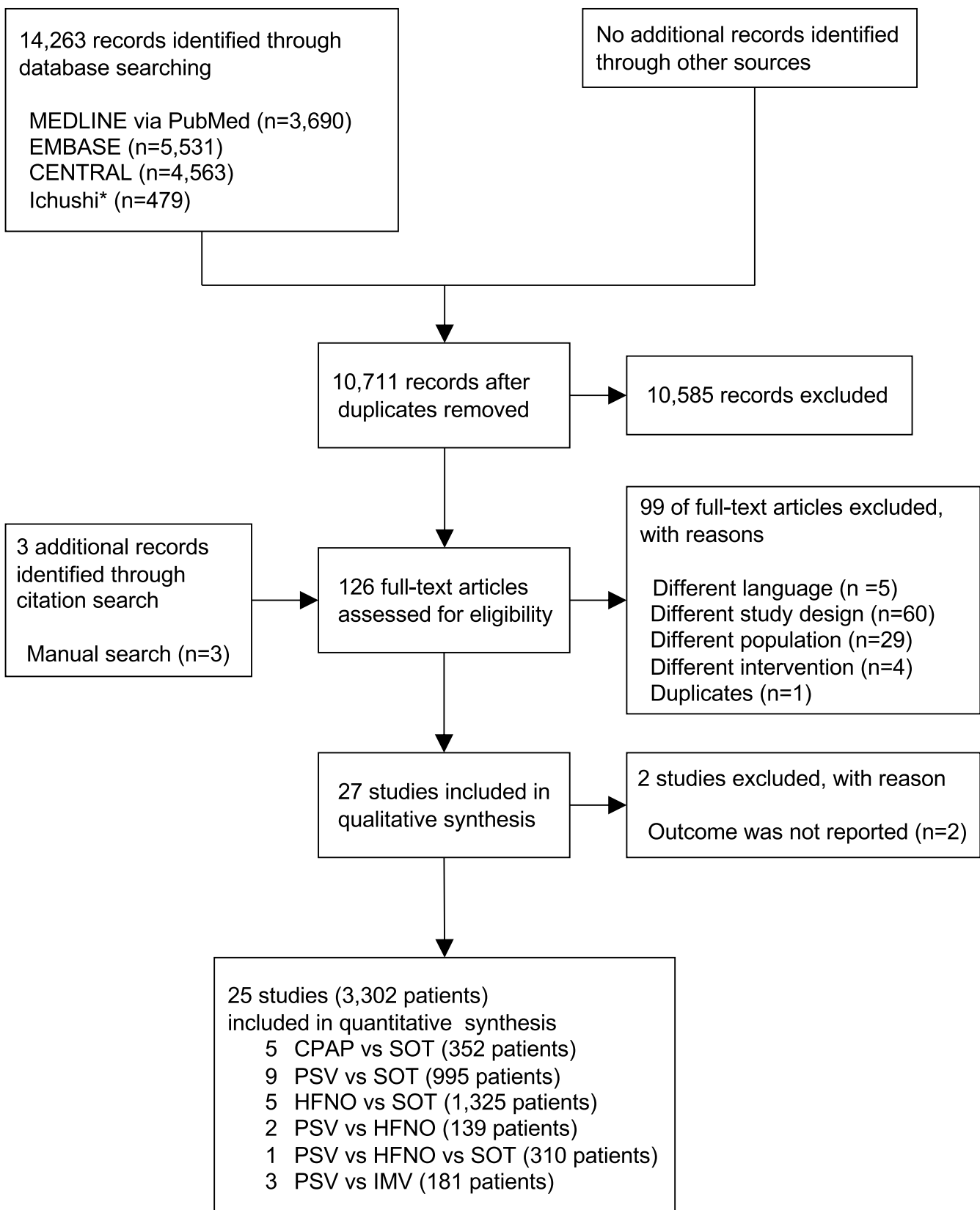

Fig. 1 Flow diagram of studies included in this review. *Ichushi is a database of Japanese research papers. CENTRAL, Cochrane Central Register of Controlled Trials; CPAP, continuous positive airway pressure; HFNO, high-flow nasal oxygen; IMV, invasive mechanical ventilation; PSV, pressure support ventilation; RCT, randomized controlled trial; RR, risk ratio; SOT, standard oxygen therapy

Non-invasive respiratory management strategies and risk of endotracheal intubation

Twenty-two trials (3,118 patients) were included in the intubation analysis. Pair-wise comparisons are shown in Additional file 1: Figure S1. The risk of bias was determined to be high for the outcome of intubation in six (27.3\%) trials (Additional file 1: Table S4). We assessed that the risk of bias was serious between CPAP and SOT and; therefore, rated down. We did not rate down due to publication bias (funnel plot shown in Additional file 1: Figure S2) and incoherence. We rated down because serious inconsistencies were observed in the comparisons of PSV vs SOT and CPAP vs SOT (Additional file 1: Table S5). 







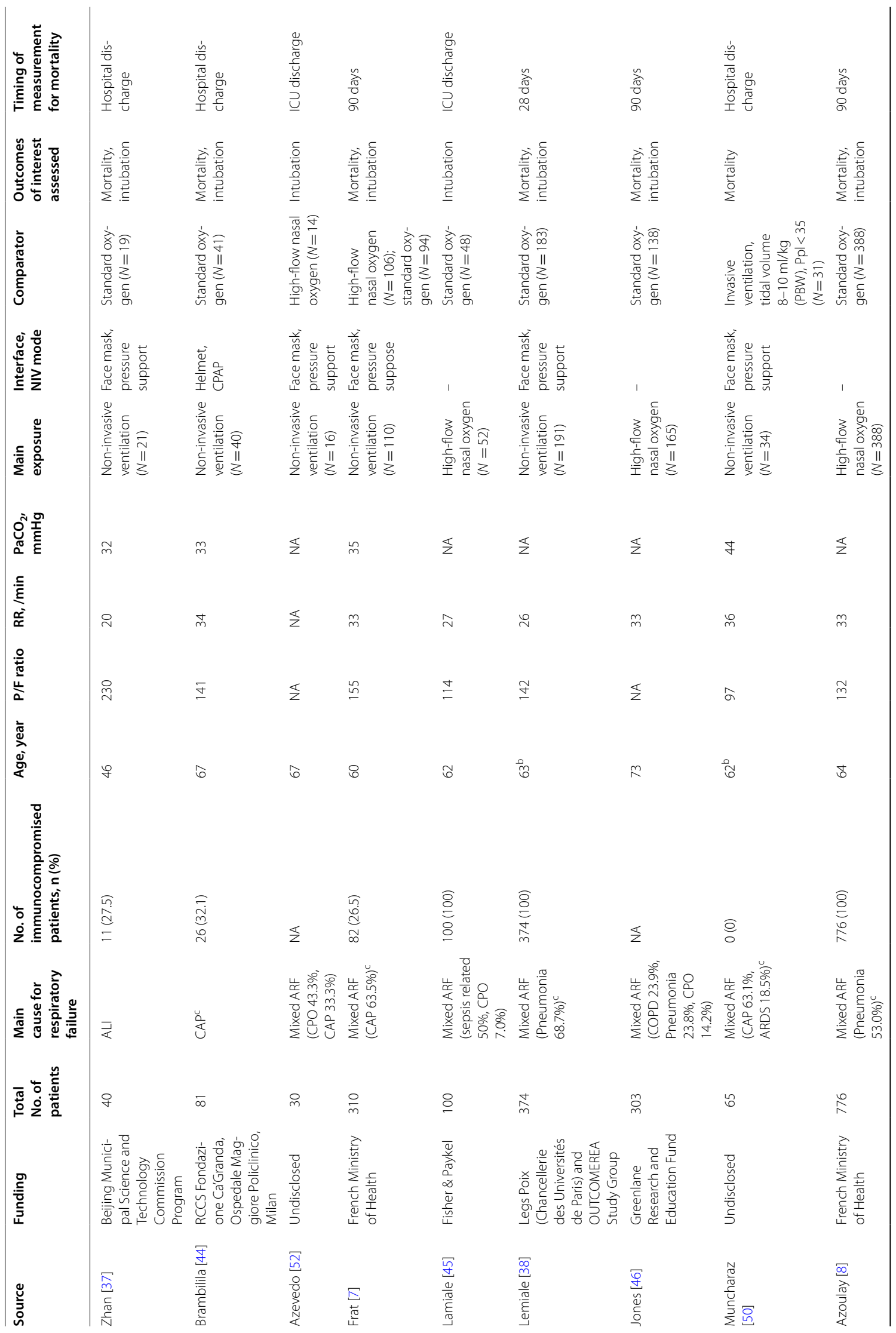




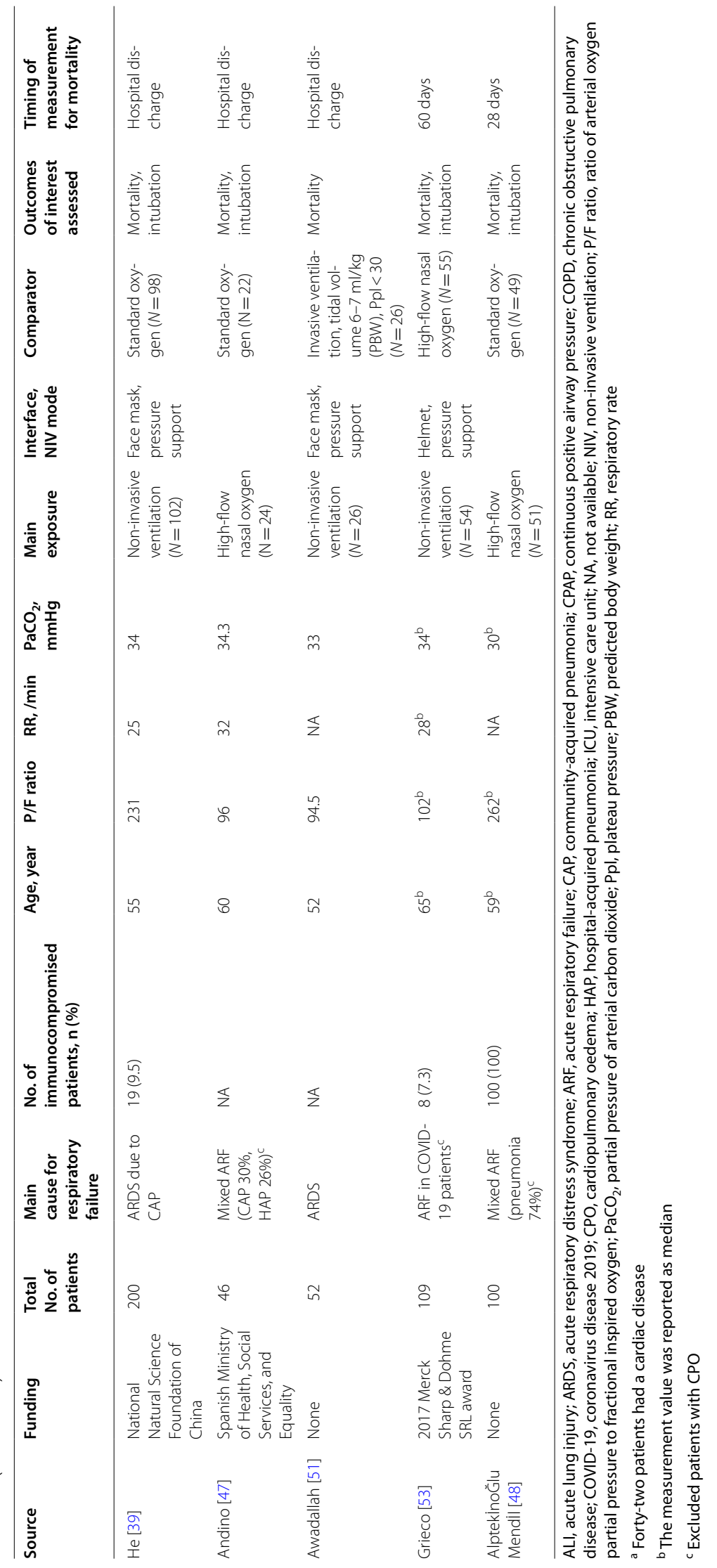


a) Short-term mortality

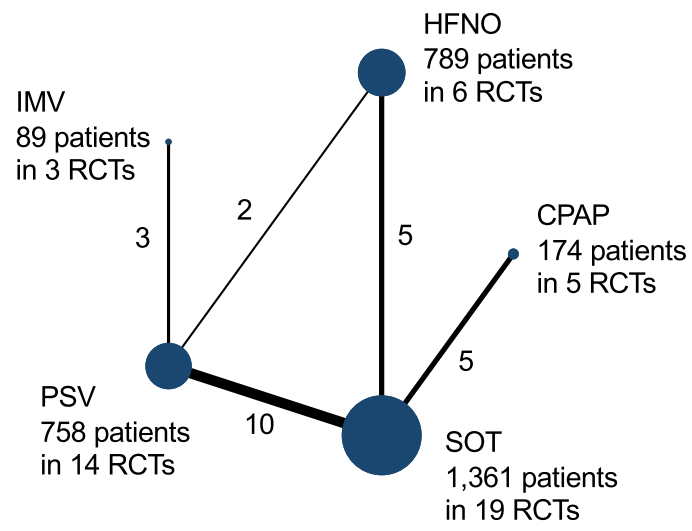

b) Endotracheal intubation

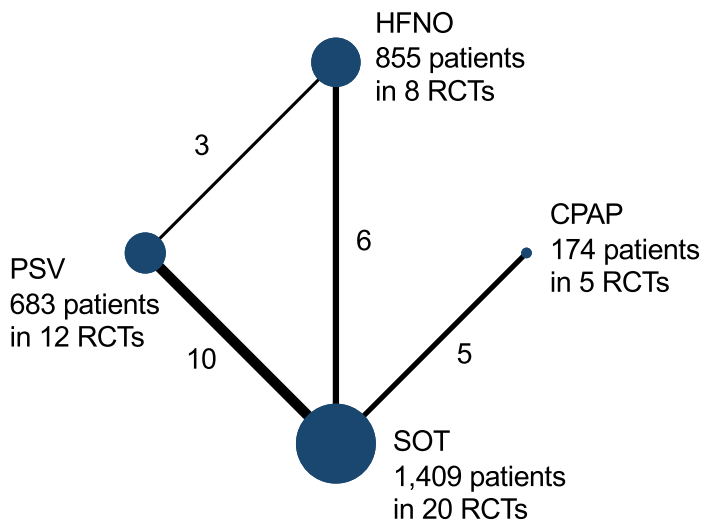

Fig. 2 Network plot for non-invasive respiratory management strategies for adults with AHRF. a For the primary outcome, short-term mortality, the longest follow-up was up to 100 days. b Secondary outcome, endotracheal intubation. When RCTs for direct comparisons exist, this is shown by connections between nodes. The size of the node represents the number of participants who received the intervention. The thickness of lines connecting nodes represents the number of trials for that comparison. CPAP, continuous positive airway pressure; HFNO, high-flow nasal oxygen; IMV, invasive mechanical ventilation; PSV, pressure support ventilation; RCT, randomized controlled trial; SOT, standard oxygen therapy

Using SOT as the reference, CPAP (RR $0.48[95 \% \mathrm{CI}$ 0.30-0.79]; RD - 0.21 [95\% CI -0.28 to -0.08 ]; low certainty) and PSV (RR 0.67 [95\% CI 0.51-0.89]; RD - 0.13 [95\% CI -0.20 to -0.04$]$; moderate certainty) were associated with a lower risk of endotracheal intubation (Fig. 3). Compared with SOT, HFNO (RR, 0.84 [95\% CI $0.61-1.17$ ]; $\mathrm{RD}-0.10$ [95\% CI -0.16 to 0.07 ]; moderate certainty) was not associated with a statistically significant lower risk of endotracheal intubation. There were no significant differences in the additional comparisons. The probability of being the best in reducing endotracheal intubation among all the possible interventions was higher for CPAP, followed by PSV, HFNO, and SOT (Table 2; Additional file 1: Figure S3).

\section{Results of additional analyses}

The results of a pre-planned sensitivity analysis excluding four studies using helmet interfaces revealed that CPAP was not associated with a lower mortality and incidence of intubation (Additional file 1: Table S6 and S7). However, for the studies comparing CPAP with SOT, which were included this analysis, there was concern with respect to the risk of bias $[40,41]$.

The results of the post hoc sensitivity analyses are shown in Additional file 1: Table S8. The observed association between CPAP and reduced risk of mortality remained significant when considering studies that included only patients with mild hypoxaemic respiratory failure (mean P/F ratio $>150$ ), and immunocompromised patients, after excluding studies with high risk of bias. On the other hand, CPAP did not show significant efficacy compared with SOT, when considering studies that included only patients with severe hypoxaemic respiratory failure (mean $\mathrm{P} / \mathrm{F}$ ratio $\leq 150$ ), and after excluding studies that enrolled any patients with COPD, cardiopulmonary oedema, or with immunocompromised status. The association of HFNO and PSV with a lower risk of mortality was not significant across almost all of the sensitivity analyses.

\section{Discussion}

Summary of evidence

In the current network meta-analyses of trials of adults with AHRF, compared with SOT, CPAP decreased the risk of death and both CPAP and PSV were associated with a lower risk of endotracheal intubation. Meanwhile, the treatment effects were not different between noninvasive respiratory management strategies and IMV for mortality. Ranking analyses showed that CPAP was the best strategy for reducing mortality and intubation. As per the results of the sensitivity analyses for mortality, CPAP also showed significant efficacy in only a few analyses, whereas compared with SOT, PSV and HFNO were not effective in almost all of the analyses.

\section{Association with previous studies}

Non-invasive ventilation is associated with a lower mortality in patients with acute respiratory failure due to cardiopulmonary oedema and COPD $[2,54]$. However, the efficacy of non-invasive respiratory management strategies in patients with de novo AHRF has been unclear [6-8]. Liu et al. [55] performed a pair-wise meta-analysis to compare the use of helmet non-invasive ventilation with control strategies, including the use of face mask 
a) Short-term mortality

\begin{tabular}{|c|c|c|c|c|c|c|c|}
\hline & $\begin{array}{l}\text { No. of } \\
\text { patients }\end{array}$ & $\begin{array}{l}\text { No. of } \\
\text { trials }\end{array}$ & Rating & $\begin{array}{c}\text { Absolute risk } \\
\text { difference }(95 \% \mathrm{Cl})\end{array}$ & $\begin{array}{l}\text { Network risk } \\
\text { ratio }(95 \% \mathrm{Cl})\end{array}$ & $\begin{array}{l}\text { Favors } \\
\text { treatment }\end{array}$ & $\begin{array}{l}\text { Favors } \\
\text { comparator }\end{array}$ \\
\hline \multicolumn{8}{|c|}{ Compared with standard oxygen } \\
\hline HFNO & 1,425 & 5 & Very low & $-0.03(-0.11$ to 0.08$)$ & $0.90(0.65-1.25)$ & & -1 \\
\hline PSV & 1,198 & 10 & Low & $-0.06(-0.11$ to 0.02$)$ & $0.81(0.62-1.06)$ & & \\
\hline CPAP & 352 & 5 & Very low & $-0.14(-0.21$ to -0.02$)$ & $0.55(0.31-0.95)$ & & \\
\hline \multicolumn{8}{|c|}{ Compared with invasive mechanical ventilation } \\
\hline HFNO & 0 & 0 & Very low & $-0.05(-0.17$ to 0.19$)$ & $0.83(0.43-1.62)$ & & \\
\hline PSV & 181 & 3 & Very low & $-0.08(-0.17$ to 0.09$)$ & $0.75(0.43-1.30)$ & & \\
\hline CPAP & 0 & 0 & Very low & $-0.15(-0.23$ to 0.05$)$ & $0.51(0.22-1.15)$ & - & -1 \\
\hline \multicolumn{8}{|c|}{ Additional comparison } \\
\hline PSV vs HFNO & 325 & 2 & Low & $-0.03(-0.11$ to 0.10$)$ & $0.90(0.62-1.32)$ & & \\
\hline CPAP vs HFNO & 0 & 0 & Very low & $-0.12(-0.20$ to 0.05$)$ & $0.61(0.32-1.15)$ & $\rightarrow$ & $\rightarrow$ \\
\hline \multirow[t]{3}{*}{ CPAP vs PSV } & 0 & 0 & Very low & $-0.10(-0.19$ to 0.07$)$ & $0.67(0.37-1.24)$ & - & $\longrightarrow$ \\
\hline & & & & & 0.2 & & 2 \\
\hline & & & & & & isk ratio (9 & $\mathrm{Cl})$ \\
\hline
\end{tabular}

b) Endotracheal intubation

\begin{tabular}{lcccccc} 
& $\begin{array}{c}\text { No. of } \\
\text { patients }\end{array}$ & $\begin{array}{c}\text { No. of } \\
\text { trials }\end{array}$ & Rating & $\begin{array}{c}\text { Absolute risk } \\
\text { difference }(95 \% \mathrm{Cl})\end{array}$ & $\begin{array}{c}\text { Network risk } \\
\text { ratio }(95 \% \mathrm{Cl})\end{array}$ \\
\cline { 1 - 5 } Compared with standard oxygen & & & & &
\end{tabular}

Fig. 3 Forest plots for association of non-invasive respiratory management strategies with study outcomes. a For the primary outcome, short-term mortality, the longest follow-up was up to 100 days. b Secondary outcome, endotracheal intubation. All outcomes are reported as network risk ratios and absolute risk differences with 95\% Cls. For estimating risk ratios for the comparison of HFNO vs IMV, CPAP vs IMV, CPAP vs HFNO, and CPAP vS PSV, only indirect evidence was used, because no direct pair-wise comparisons were available. The estimated absolute risks of mortality and endotracheal intubation were $30 \%$ and $40 \%$, respectively, in the control group. Cl, confidence interval; CPAP, continuous positive airway pressure; HFNO, high-flow nasal oxygen; IMV, invasive mechanical ventilation; PSV, pressure support ventilation; RR, risk ratio; SOT, standard oxygen therapy

non-invasive ventilation and SOT, and demonstrated that helmet non-invasive ventilation was associated with reduced hospital mortality and intubation requirement. Although both CPAP and PSV showed significant benefit in the subgroup analyses, six of eight studies using PSV were conducted among patients with acute exacerbation of COPD. The efficacy of non-invasive ventilation according to ventilation modes could not be evaluated in patients with AHRF. In 2020, Ferreyro et al. [10] reported an NMA in which the efficacy of non-invasive respiratory management strategies were compared with that of SOT among adult patients with AHRF and found that helmet non-invasive ventilation was associated with a lower risk of mortality and intubation compared with SOT, HFNO, and face mask non-invasive ventilation. However, the NMA included patients with postoperative respiratory failure or chest trauma. Those patients had various causes of respiratory failure, including atelectasis due to poor pain control, chest wall injury, and pleural effusion, not only because of lung injury. We included trials in which more than half of the patients were experiencing de novo AHRF. Although the cause of AHRF was still 
Table 2 Results of network rank test in the Network Meta-analysis

\begin{tabular}{|c|c|c|c|c|c|c|}
\hline & CPAP & PSV & & HFNO & IMV & SOT \\
\hline \multicolumn{7}{|c|}{ a. Short-term mortality } \\
\hline Best & $84.0 \%$ & $8.1 \%$ & & $3.8 \%$ & $4.0 \%$ & $0.1 \%$ \\
\hline 2nd & $9.0 \%$ & $52.6 \%$ & & $23.3 \%$ & $12.1 \%$ & $3.0 \%$ \\
\hline $3 \mathrm{rd}$ & $3.9 \%$ & $31.4 \%$ & & $32.1 \%$ & $13.6 \%$ & $19.0 \%$ \\
\hline 4th & $1.9 \%$ & $7.1 \%$ & & $26.9 \%$ & $16.0 \%$ & $48.1 \%$ \\
\hline Worst & $1.2 \%$ & $0.8 \%$ & & $13.9 \%$ & $54.3 \%$ & $29.8 \%$ \\
\hline Mean rank & 1.3 & 2.4 & & 3.2 & 4.0 & 4.0 \\
\hline \multirow[t]{2}{*}{ SUCRA } & 93.2 & 65.0 & & 44.1 & 23.9 & 23.9 \\
\hline & CPAP & & PSV & \multicolumn{2}{|r|}{ HFNO } & SOT \\
\hline \multicolumn{7}{|c|}{ b. Endotracheal intubation } \\
\hline Best & \multicolumn{2}{|l|}{$88.5 \%$} & \multicolumn{2}{|l|}{$10.8 \%$} & $0.7 \%$ & $0.0 \%$ \\
\hline $2 n d$ & \multicolumn{2}{|l|}{$8.8 \%$} & \multicolumn{2}{|l|}{$79.0 \%$} & $12.1 \%$ & $0.1 \%$ \\
\hline $3 r d$ & \multicolumn{2}{|l|}{$2.6 \%$} & \multicolumn{2}{|l|}{$10.0 \%$} & $74.2 \%$ & $13.2 \%$ \\
\hline Worst & \multicolumn{2}{|l|}{$0.1 \%$} & \multicolumn{2}{|l|}{$0.2 \%$} & $13.0 \%$ & $86.7 \%$ \\
\hline Mean rank & 1.1 & & \multicolumn{2}{|l|}{2.0} & 3.0 & 3.9 \\
\hline SUCRA & 95.2 & & \multicolumn{2}{|l|}{66.8} & 33.5 & 4.5 \\
\hline
\end{tabular}

CPAP, continuous positive airway pressure; HFNO, high-flow nasal oxygenation; IMV, invasive mechanical ventilation; PSV, pressure support ventilation; SCURA, surface under the cumulative ranking; SOT, standard oxygen therapy

${ }^{a}$ For the primary outcome, short-term mortality, the longest follow-up was up to 100 days

${ }^{b}$ For the secondary outcome, endotracheal intubation

inconsistent, our analysis included a higher proportion of patients with de novo AHRF compared with the previous NMA.

There were insufficient data examining HFNO compared to non-invasive ventilation among patients with de novo AHRF. As per the results from an RCT comparing the use of helmet PSV with HFNO in patients with AHRF due to the coronavirus disease 2019, helmet PSV was associated with a higher $\mathrm{P} / \mathrm{F}$ ratio and $\mathrm{PaCO}_{2}$ [53]. Although the rate of intubation was lower in patients with the use of a helmet PSV, the mortality was not different. A helmet interface can decrease air leaks and provide higher levels of PEEP, potentially increasing alveolar recruitment and improving oxygenation [56], but increasing dead space may worsen ventilation and contribute to lager tidal volume. Although the PEEP effect of HFNO may not be sufficient to avoid intubation, it is unclear which is a better strategy, HFNO or non-invasive ventilation, considering dead space. In our NMA, HFNO did not show a reduction in the rate of mortality and incidence of intubation compared with other respiratory management strategies. Further evaluation is needed to provide conclusive recommendations, although HFNO was recommended for patients with AHRF compared with SOT [3].

In all trials comparing helmet non-invasive ventilation with SOT, which were included in the previous NMA, CPAP was used as a ventilation mode [10]. The use of
CPAP might contribute to the superiority of helmet noninvasive ventilation. According to an RCT comparing helmet interface to face mask in patients who underwent non-invasive ventilation, patients with a helmet interface were set at a lower level of pressure support and had a lower mortality [57]. Since excessive tidal volume may worsen outcomes [9], it may be important to set lower levels of pressure support for patients with AHRF. CPAP also has advantages over non-invasive ventilation in terms of having simpler technology, better synchrony, and requiring potentially less expensive equipment [6]. Our findings imply that CPAP is the most effective among the non-invasive respiratory management strategies, in concordance with these physiological effects.

\section{Significance and implications}

A high respiratory drive and large tidal volume may contribute to patient self-inflicted lung injury and poor outcomes in patients with AHRF [58-60]. In our NMA, PSV was not associated with lower mortality, but CPAP decreased mortality and the incidence of endotracheal intubation compared with SOT. Furthermore, ranking analyses showed that CPAP was the best strategy for reducing mortality and intubation. Normally, when CPAP is used as a primary ventilation mode, we do not use pressure support unless pressure support is needed (e.g. in patients with hypercapnia, those with a lack of tidal volume, and those with a high respiratory drive). It 
may contribute the reduction of using unnecessary pressure support. When performing non-invasive ventilation in patients with AHRF, PEEP recruiting the lungs and maintaining them open may reduce respiratory drive and contribute to lung protection. Although pressure support is needed for some patients with AHRF, we should use pressure support with caution as this may lead to excessive tidal volume and lung injury. An ongoing RCT (jRCTs052180236) may provide further evidence to support these claims.

Although non-invasive ventilation is performed to avoid intubation, treatment failure has been reported to occur in $37.5 \%$ of patients with AHRF [4]. Furthermore, treatment failure has been associated with hospital mortality. De novo AHRF, including ARDS, was one of the risk factors for non-invasive ventilation failure [61]. Despite the high risk of treatment failure, no metaanalyses have been reported to compare non-invasive respiratory management strategies with IMV. We did not find significant differences between non-invasive respiratory management strategies and IMV, which was not considered as lung-protective ventilation in most of the included trials, in a decrease of mortality. It remains unclear whether it is better to ensure lung protection or avoid complications of endotracheal intubation. CPAP demonstrated the efficacy in the sensitivity analysis among patients with mild hypoxaemia, but not with severe hypoxaemia. Since lung-protective ventilation using neuromuscular blockers is recommended strongly in patients with severe hypoxaemia [62], our findings imply that non-invasive management strategies should not be performed in such patients.

\section{Strengths and limitations}

To the best of our knowledge, no systematic reviews and meta-analyses have been performed to evaluate noninvasive ventilation according to ventilation modes and compare them with IMV in adults with AHRF. However, the current NMA also had several limitations. First, language restrictions may have contributed to the inclusion of an inadequate number of studies. However, we did not identify any trials in other languages being included in previous meta-analyses that had no language restrictions $[10,63]$. Therefore, we believe that the language restriction had no effect. Second, we included studies with patients with cardiopulmonary oedema and COPD who were at a low risk of non-invasive ventilation failure. This may contribute to overestimating the treatment effect. The NMA assumption was that individual trials enrolled similar populations, and that the intervention protocol was similar across the different studies. We need to interpret results from the current network meta-analysis with caution because of a variety of causes leading to AHRF. Similar to the results from a post hoc analysis that excluded patients with cardiopulmonary oedema and COPD, we did not find any significant efficacy with the implementation of non-invasive respiratory management strategies. Our findings did not provide conclusive evidence among patients with de novo AHRF. Third, the effect of non-invasive ventilation may not have been consistent with respect to patient severity [4]. The mean P/F ratio in studies in which IMV was compared was lower than that in studies in which SOT was compared. The differences in the treatment effect may affect intransitivity and incoherence in an NMA. Fourth, there was a concern about the primary studies included in our review regarding the lack of blinding of the treatment groups. Although this was unlikely to bias the assessment of hard outcomes, it may have contributed to performance bias. Fifth, we did not observe a significant benefit with CPAP in only a few sensitivity analyses. In contrast, PSV and HFNO were not effective in almost all of the analyses compared with SOT. Further studies evaluating CPAP with more participants are needed to provide robust evidence because most trials had a small sample size. Sixth, network RR was only estimated by indirect evidence in some comparisons. Specifically, few studies compared non-invasive respiratory management strategies with IMV. Further studies are needed to provide a higher certainty of evidence. Seventh, most studies did not report on tidal volume with predicted body weight. Thus, it was unclear whether pressure support was associated with larger tidal volume. Finally, the fact that the included studies reported different follow-up times for all-cause mortality may have contributed to the heterogeneity. However, as per the results of the sensitivity analyses, the effect on mortality within 30 days was similar to that from the main analysis.

\section{Conclusions}

The current network meta-analysis demonstrated that CPAP may be the most effective respiratory management strategy among patients with AHRF. Considering the low certainty of the current evidence, particularly compared with IMV, further studies are required to clarify whether non-invasive respiratory management strategies for de novo AHRF are effective or not. When performing noninvasive ventilation among patients with de novo AHRF, it is important to avoid excessive tidal volume and lung injury. Although pressure support is needed for some of these patients, it should be applied with caution because this may lead to excessive tidal volume and lung injury. If the risk of lung injury cannot be avoided, we should ensure lung-protective ventilation with endotracheal intubation, especially in patients with severe hypoxaemia. 


\begin{abstract}
Abbreviations
AHRF: Acute hypoxemic respiratory failure; ALI: Acute lung injury; ARDS: Acute respiratory distress syndrome; ARF: Acute respiratory failure; CAP: Communityacquired pneumonia; Cl: Confidence interval; COPD: Chronic obstructive pulmonary disease; COVID-19: Coronavirus disease 2019; CPAP: Continuous positive airway pressure; GRADE: Grades of Recommendation, Assessment, Development and Evaluation Working Group; HFNO: High-flow nasal oxygen; ICU: Intensive care unit; IMV: Invasive mechanical ventilation; NMA: Network meta-analysis; PSV: Pressure support ventilation; P/F ratio: Ratio of arterial oxygen partial pressure to fractional inspired oxygen; $\mathrm{PaCO}_{2}$ : Partial pressure of arterial carbon dioxide; $\mathrm{PaO}_{2}$ : Partial pressure of arterial oxygen; $\mathrm{PBW}$ : Predicted body weight; PEEP: Positive end-expiratory pressure; RCT: Randomized controlled trial; RevMan: Review manager; RR: Risk ratio; RD: Risk difference; SOT: Standard oxygen therapy; SUCRA: Surface under the cumulative ranking curve.
\end{abstract}

\section{Supplementary Information}

The online version contains supplementary material available at https://doi. org/10.1186/s13054-021-03835-8.

Additional file 1. Table S1. PRISMA NMA checklist. Table S2. Search strategy. Table S3. The proportion of patients with cause of respiratory failure or situation for exclusion criteria. Table $\mathbf{S 4}$. Summary of risk of bias of the studies included in the network meta-analysis. Table $\mathbf{S 5}$. Summary of network meta-analysis and GRADE assessment for the effects of non-invasive respiratory management strategies. Table S6. Pre-planned sensitivity analysis for the effect of non-invasive respiratory management strategies on outcomes in the network meta-analysis (excluding studies with helmet interfaces). Table S7. Pre-planned sensitivity analyses for the network rank test in the network meta-analysis (excluding studies with helmet interfaces). Table S8 Post-hoc sensitivity analyses for the effect of noninvasive respiratory management strategies on short-term mortality in the network meta-analysis. Fig. S1. Summary of random effects meta-analysis for direct comparisons of non-invasive respiratory management strategies for adults with AHRF (RevMan 5.3). Fig. S2. Comparison adjusted funnel plot for the network meta-analysis. Fig. S3. Results of ranking probability in the network meta-analysis.

\section{Acknowledgements}

We would thank all the members of the Japanese ARDS clinical practice guideline committee from the Japanese Society of Respiratory Care Medicine, the Japanese Society of Respiratory and the Japanese Society of Intensive Care Medicine. We also appreciate the librarian Takaaki Suzuki at the Nara Medical University Library, the librarians at the Kyoto Prefectural University of Medicine for supporting our search strategies, and Editage (www.editage. com) for English language editing.

\section{Authors' contributions}

MS designed the study, acquired data, performed statistical analyses, and interpreted the data. $\mathrm{HO}$ and $\mathrm{TM}$ conceived the study and acquired and interpreted the data. SK and SH conceived the acquisition of data. The first draft of the manuscript was written by MS, and all authors commented on previous versions of the manuscript. All authors read and approved the final manuscript.

\section{Funding}

Not applicable.

\section{Availability of data and materials}

The datasets used and analysed during the current network meta-analysis are available from the corresponding author upon reasonable request.

\section{Declarations}

Ethics approval and consent to participate Not applicable.

\section{Consent for publication}

Not applicable.

\section{Competing interests}

All the authors declare that they have no conflicts of interest.

\section{Author details}

${ }^{1}$ Department of Emergency and Intensive Care Medicine, JA Hiroshima General Hospital, Jigozen 1-3-3, Hatsukaichi, Hiroshima 738-8503, Japan. ${ }^{2}$ Department of Critical and Emergency Medicine, National Hospital Organization Yokohama Medical, Yokohama, Japan. ${ }^{3}$ Department of Emergency and Critical Care Medicine, Misato Kenwa Hospital, Saitama, Japan. ${ }^{4}$ Department of Preventive Services, School of Public Health, Kyoto University, Kyoto, Japan. ${ }^{5}$ Department of Respiratory Medicine and Infectious Diseases, Niigata University Graduate School of Medical and Dental Sciences, Niigata, Japan.

Received: 25 August 2021 Accepted: 20 November 2021

Published online: 29 November 2021

\section{References}

1. Vincent J-L, Akça S, de Mendonça A, Haji-Michael P, Sprung C, Moreno R, et al. The epidemiology of acute respiratory failure in critically ill patients. Chest. 2002;12:1602-9.

2. Berbenetz N, Wang Y, Brown J, Godfrey C, Ahmad M, Vital FM, et al. Non-invasive positive pressure ventilation (CPAP or bilevel NPPV) for cardiogenic pulmonary oedema. Cochrane Database Syst Rev. 2019:4:Cd005351.

3. Rochwerg B, Einav S, Chaudhuri D, Mancebo J, Mauri T, Helviz Y, et al. The role for high flow nasal cannula as a respiratory support strategy in adults: a clinical practice guideline. Intensive Care Med. 2020;46:2226-37.

4. Bellani G, Laffey JG, Pham T, Madotto F, Fan E, Brochard L, et al. Noninvasive ventilation of patients with acute respiratory distress syndrome. insights from the LUNG SAFE study. Am J Respir Crit Care Med. 2017;195:67-77.

5. Ranieri VM, Rubenfeld GD, Thompson BT, Ferguson ND, Caldwell E, Fan $E$, et al. Acute respiratory distress syndrome: the Berlin Definition. JAMA. 2012;307:2526-33.

6. Rochwerg B, Brochard L, Elliott MW, Hess D, Hill NS, Nava S, et al. Official ERS/ATS clinical practice guidelines: noninvasive ventilation for acute respiratory failure. Eur Respir J. 2017;50:1602426.

7. Frat JP, Thille AW, Mercat A, Girault C, Ragot S, Perbet S, et al. High-flow oxygen through nasal cannula in acute hypoxemic respiratory failure. $\mathrm{N}$ Engl J Med. 2015;372:2185-96.

8. Azoulay E, Lemiale V, Mokart D, Nseir S, Argaud L, Pène F, et al. Effect of high-flow nasal oxygen vs standard oxygen on 28-day mortality in immunocompromised patients with acute respiratory failure: the $\mathrm{HIGH}$ randomized clinical trial. JAMA. 2018;320:2099-107.

9. Carteaux G, Millán-Guilarte T, De Prost N, Razazi K, Abid S, Thille AW, et al. Failure of noninvasive ventilation for de novo acute hypoxemic respiratory failure: role of tidal volume. Crit Care Med. 2016;44:282-90.

10. Ferreyro BL, Angriman F, Munshi L, Del Sorbo L, Ferguson ND, Rochwerg $B$, et al. Association of noninvasive oxygenation strategies with all-cause mortality in adults with acute hypoxemic respiratory failure: a systematic review and meta-analysis. JAMA. 2020;324:57-67.

11. Hutton B, Salanti G, Caldwell DM, Chaimani A, Schmid CH, Cameron C, et al. The PRISMA extension statement for reporting of systematic reviews incorporating network meta-analyses of health care interventions: checklist and explanations. Ann Intern Med. 2015;62:777-84.

12. Sakuraya M, Okano H, Masuyama T, Kimata S, Hokari S. Which NPPV mode, CPAP or PSV, is the best solution for the management of acute hypoxemic respiratory failure: a systematic review and network metaanalysis protocol. 2021; https://doi.org/10.17504/protocols.io.buf7ntrn. Accessed 24 Apr 2021.

13. Keenan SP, Sinuff T, Burns KE, Muscedere J, Kutsogiannis J, Mehta S, et al. Clinical practice guidelines for the use of noninvasive positive-pressure ventilation and noninvasive continuous positive airway pressure in the acute care setting. CMAJ. 2011;183:E195-214. 
14. The Cochrane Collaboration. Data collection form-intervention review for RCTs only. Secondary data collection form-intervention review for RCTs only. 2014. https://dplp.cochrane.org/data-extraction-forms. Accessed 23 Apr 2021.

15. The Cochrane Collaboration. RevMan 5 Download and installation. Secondary RevMan 5 download and installation. 2014. https://community. cochrane.org/help/tools-and-software/revman-5/revman-5-download/ installation. Accessed 23 Apr 2021.

16. Sterne JAC, Savović J, Page MJ, Elbers RG, Blencowe NS, Boutron I, et al. RoB 2: a revised tool for assessing risk of bias in randomised trials. BMJ. 2019:366:14898.

17. White IR, Barrett JK, Jackson D, Higgins JP. Consistency and inconsistency in network meta-analysis: model estimation using multivariate metaregression. Res Synth Methods. 2012;3:111-25.

18. Salanti G, Ades AE, loannidis JPA. Graphical methods and numerical summaries for presenting results from multiple-treatment meta-analysis: an overview and tutorial. J Clin Epidemiol. 2011;64:163-71.

19. Chaimani A, Higgins JP, Mavridis D, Spyridonos P, Salanti G. Graphical tools for network meta-analysis in STATA. PLOS ONE. 2013;8:e76654.

20. Higgins JP, Thompson SG. Quantifying heterogeneity in a meta-analysis. Stat Med. 2002;21:1539-58.

21. Lumley T. Network meta-analysis for indirect treatment comparisons. Stat Med. 2002;21:2313-24.

22. Dias S, Welton NJ, Caldwell DM, Ades AE. Checking consistency in mixed treatment comparison meta-analysis. Stat Med. 2010;29:932-44.

23. Guyatt GH, Oxman AD, Kunz R, Woodcock J, Brozek J, Helfand M, et al. GRADE guidelines: 7-rating the quality of evidence-inconsistency. J Clin Epidemiol. 2011;64:1294-302.

24. Guyatt GH, Oxman AD, Montori V, Vist G, Kunz R, Brozek J, et al. GRADE guidelines: 5-rating the quality of evidence-publication bias. J Clin Epidemiol. 2011;64:1277-82

25. Guyatt GH, Oxman AD, Vist G, Kunz R, Brozek J, Alonso-Coello P, et al. GRADE guidelines: 4-rating the quality of evidence-study limitations (risk of bias). J Clin Epidemiol. 2011;64:407-15.

26. Puhan MA, Schünemann HJ, Murad MH, Li T, Brignardello-Petersen $R$, Singh JA, et al. A GRADE Working Group approach for rating the quality of treatment effect estimates from network meta-analysis. BMJ. 2014;349:g5630.

27. Brignardello-Petersen $R$, Bonner A, Alexander PE, Siemieniuk RA, Furukawa TA, Rochwerg B, et al. Advances in the GRADE approach to rate the certainty in estimates from a network meta-analysis. J Clin Epidemiol. 2018:93:36-44.

28. American College of Chest Physicians/Society of Critical Care Medicine Consensus Conference: definitions for sepsis and organ failure and guidelines for the use of innovative therapies in sepsis. Crit Care Med. 1992:20:864-74.

29. Guyatt GH, Oxman AD, Kunz R, Brozek J, Alonso-Coello P, Rind D, et al. GRADE guidelines 6-rating the quality of evidence-imprecision. J Clin Epidemiol. 2011;64:1283-93.

30. Brignardello-Petersen R, Guyatt GH, Mustafa RA, Chu DK, Hultcrantz M, Schünemann $\mathrm{HJ}$, et al. GRADE guidelines 33-addressing imprecision in a network meta-analysis. J Clin Epidemiol. 2021;139:49-56.

31. Wysocki M, Tric L, Wolff MA, Millet H, Herman B. Noninvasive pressure support ventilation in patients with acute respiratory failure-a randomized comparison with conventional therapy. Chest. 1995;107:761-8.

32. Confalonieri M, Potena A, Carbone G, Porta RD, Tolley EA, Umberto MG. Acute respiratory failure in patients with severe community-acquired pneumonia: a prospective randomized evaluation of noninvasive ventilation. Am J Respir Crit Care Med. 1999;160:1585-91.

33. Antonelli M, Conti G, Bufi M, Costa MG, Lappa A, Rocco M, et al. Noninvasive ventilation for treatment of acute respiratory failure in patients undergoing solid organ transplantation: a randomized trial. JAMA. 2000;283:235-41.

34. Hilbert G, Gruson D, Vargas F, Valentino R, Gbikpi-Benissan G, Dupon $M$, et al. Noninvasive ventilation in immunosuppressed patients with pulmonary infiltrates, fever, and acute respiratory failure. N Engl J Med. 2001;344:481-7.

35. Ferrer M, Esquinas A, Leon M, Gonzalez G, Alarcon A, Torres A. Noninvasive ventilation in severe hypoxemic respiratory failure: a randomized clinical trial. Am J Respir Crit Care Med. 2003;168:1438-44.
36. Wermke M, Schiemanck S, Höffken G, Ehninger G, Bornhäuser M, IIlmer T. Respiratory failure in patients undergoing allogeneic hematopoietic SCT-a randomized trial on early non-invasive ventilation based on standard care hematology wards. Bone Marrow Transplant. 2012;47:574-80.

37. Zhan Q, Sun B, Liang L, Yan X, Zhang L, Yang J, et al. Early use of noninvasive positive pressure ventilation for acute lung injury: a multicenter randomized controlled trial. Crit Care Med. 2012;40:455-60.

38. Lemiale V, Mokart D, Resche-Rigon M, Pène F, Mayaux J, Faucher E, Nyunga $\mathrm{M}$, et al. Effect of noninvasive ventilation vs oxygen therapy on mortality among immunocompromised patients with acute respiratory failure: a randomized clinical trial. JAMA. 2015;314:1711-9.

39. He H, Sun B, Liang L, Li Y, Wang H, Wei L, et al. A multicenter RCT of noninvasive ventilation in pneumonia-induced early mild acute respiratory distress syndrome. Crit Care. 2019;23:300.

40. Delclaux C, L'Her E, Alberti C, Mancebo J, Abroug F, Conti G, et al. Treatment of acute hypoxemic nonhypercapnic respiratory insufficiency with continuous positive airway pressure delivered by a face mask: a randomized controlled trial. JAMA. 2000;284:2352-60.

41. Martin TJ, Hovis JD, Costantino JP, Bierman MI, Donahoe MP, Rogers RM, et al. A randomized, prospective evaluation of noninvasive ventilation for acute respiratory failure. Am J Respir Crit Care Med. 2000;161:807-13.

42. Cosentini R, Brambilla AM, Aliberti S, Bignamini A, Nava S, Maffei A, et al. Helmet continuous positive airway pressure vs oxygen therapy to improve oxygenation in community-acquired pneumonia: a randomized, controlled trial. Chest. 2010;138:114-20.

43. Squadrone V, Massaia M, Bruno B, Marmont F, Falda M, Bagna C, et al. Early CPAP prevents evolution of acute lung injury in patients with hematologic malignancy. Intensive Care Med. 2010;36:1666-74.

44. Brambilla AM, Aliberti S, Prina E, Nicoli F, Del Forno M, Nava S, et al. Helmet CPAP vs oxygen therapy in severe hypoxemic respiratory failure due to pneumonia. Intensive Care Med. 2014;40:942-9.

45. Lemiale V, Mokart D, Mayaux J, Lambert J, Rabbat A, Demoule A, et al. The effects of a 2-h trial of high-flow oxygen by nasal cannula versus Venturi mask in immunocompromised patients with hypoxemic acute respiratory failure: a multicenter randomized trial. Crit Care. 2015;19:380.

46. Jones PG, Kamona S, Doran O, Sawtell F, Wilsher M. Randomized controlled trial of humidified high-flow nasal oxygen for acute respiratory distress in the emergency department: the HOT-ER study. Respir Care. 2016;61:291-9.

47. Andino R, Vega G, Pacheco SK, Arevalillo N, Leal A, Fernández L, et al. High-flow nasal oxygen reduces endotracheal intubation: a randomized clinical trial. Ther Adv Respir Dis. 2020:14:1753466620956459.

48. AlpteklnoĞlu Mendill N, Temel Ş, YÜksel RC, GÜndoĞan K, Eser B, Kaynar $L$, et al. The use of high-flow nasal oxygen vs standard oxygen therapy in hematological malignancy patients with acute respiratory failure in hematology wards. Turk J Med Sci. 2021. https://doi.org/10.3906/ sag-2007-228.

49. Antonelli M, Conti G, Rocco M, Bufi M, De Blasi RA, Vivino G, et al. A comparison of noninvasive positive-pressure ventilation and conventional mechanical ventilation in patients with acute respiratory failure. N Engl J Med. 1998;339:429-35.

50. Muncharaz AB, Bort MC, Asensio DB, Campos LM, Tegedor BV, Pérez JM, et al. Non-invasive ventilation versus invasive mechanical ventilation in patients with hypoxemic acute respiratory failure in an intensive care unit—a randomized controlled study. Minerva Pneumol. 2017;56:1-10.

51. Awadallah M, Taha A, Sarhan T. Cardiorespiratory changes and outcome during noninvasive and invasive mechanical ventilation in ARDS: a comparative study. Res Opin Anesthes Intensive Care. 2021;8:6-12.

52. Azevedo JR, Montenegro WS, Leitao AL, Silva MM, Prazeres JS, Maranhao JP. High flow nasal cannula oxygen (hfnc) versus non-invasive positive pressure ventilation (nippv) in acute hypoxemic respiratory failure-a pilot randomized controlled trial [abstract]. Intensive Care Med Exp. 2015;3(Suppl 1):A166.

53. Grieco DL, Menga LS, Cesarano M, Rosà T, Spadaro S, Bitondo MM, et al. Effect of helmet noninvasive ventilation vs high-flow nasal oxygen on days free of respiratory support in patients with COVID-19 and moderate to severe hypoxemic respiratory failure: the HENIVOT randomized clinical trial. JAMA. 2021;325:1731-43.

54. Osadnik CR, Tee VS, Carson-Chahhoud KV, Picot J, Wedzicha JA, Smith BJ. Non-invasive ventilation for the management of acute hypercapnic 
respiratory failure due to exacerbation of chronic obstructive pulmonary disease. Cochrane Database Syst Rev. 2017;7:Cd004104.

55. Liu Q, Gao Y, Chen R, Cheng Z. Noninvasive ventilation with helmet versus control strategy in patients with acute respiratory failure: a systematic review and meta-analysis of controlled studies. Crit Care. 2016;20:265.

56. Esquinas Rodriguez AM, Papadakos PJ, Carron M, Cosentini R, Chiumello D. Clinical review: Helmet and non-invasive mechanical ventilation in critically ill patients. Crit Care. 2013;17:223.

57. Patel BK, Wolfe KS, Pohlman AS, Hall JB, Kress JP. Effect of noninvasive ventilation delivered by helmet vs face mask on the rate of endotracheal intubation in patients with acute respiratory distress syndrome: a randomized clinical trial. JAMA. 2016;315:2435-41.

58. Slutsky AS, Ranieri VM. Ventilator-induced lung injury. N Engl J Med. 2013;369:2126-36.

59. Brochard L, Slutsky A, Pesenti A. Mechanical ventilation to minimize progression of lung injury in acute respiratory failure. Am J Respir Crit Care Med. 2017;195:438-42.
60. Onelli R, Fantini R, Tabbì L, Castaniere I, Pisani L, Pellegrino MR, et al. Early inspiratory effort assessment by oesophageal manometry predicts noninvasive ventilation outcome in de novo respiratory failure: a pilot study. Am J Respir Crit Care Med. 2020;202:558-67.

61. Scala R, Pisani L. Noninvasive ventilation in acute respiratory failure: which recipe for success? Eur Respir Rev. 2018;27:180029.

62. Papazian L, Forel JM, Gacouin A, Penot-Ragon C, Perrin G, Loundou A, et al. Neuromuscular blockers in early acute respiratory distress syndrome. N Engl J Med. 2010;363:1107-16.

63. Zayed Y, Barbarawi M, Kheiri B, Haykal T, Chahine A, Rashdan L, et al. Initial noninvasive oxygenation strategies in subjects with de novo acute hypoxemic respiratory failure. Respir Care. 2019;64:433-44.

\section{Publisher's Note}

Springer Nature remains neutral with regard to jurisdictional claims in published maps and institutional affiliations.
Ready to submit your research? Choose BMC and benefit from:

- fast, convenient online submission

- thorough peer review by experienced researchers in your field

- rapid publication on acceptance

- support for research data, including large and complex data types

- gold Open Access which fosters wider collaboration and increased citations

- maximum visibility for your research: over $100 \mathrm{M}$ website views per year

At BMC, research is always in progress.

Learn more biomedcentral.com/submissions 